\title{
Belphégor
}

\section{Fantômas a cent ans}

\section{Robin Walz et Sándor Kalai}

\section{(2) OpenEdition}

\section{Journals}

Édition électronique

URL : http://journals.openedition.org/belphegor/153

DOI : 10.4000/belphegor.153

ISSN : 1499-7185

Éditeur

LPCM

Référence électronique

Robin Walz et Sándor Kalai, « Fantômas a cent ans », Belphégor [En ligne], 11-1 | 2013, mis en ligne le 01 mai 2013, consulté le 22 septembre 2020. URL : http://journals.openedition.org/belphegor/153 DOI : https://doi.org/10.4000/belphegor.153

Ce document a été généré automatiquement le 22 septembre 2020.

\section{(c)}

Belphégor est mis à disposition selon les termes de la Licence Creative Commons Attribution - Pas d'Utilisation Commerciale - Pas de Modification 4.0 International. 


\title{
Fantômas a cent ans
}

\author{
Robin Walz et Sándor Kalai
}

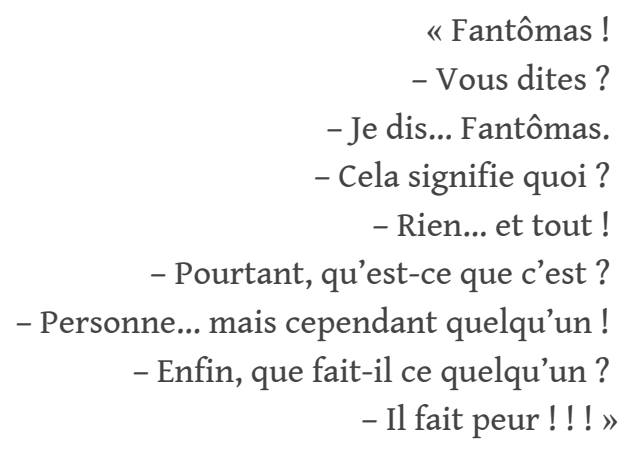

C'est il y a un siècle que l'ombre immense de Fantômas s'est allongée pour la première fois sur Paris. En février 1911 ce dialogue préliminaire assez opaque tentant de saisir l'Insaisissable a provoqué chez les lecteurs des frissons d'inquiétude (et aussi, sans doute, de plaisir). Mais aujourd'hui le sens de ces phrases continue d'être aussi énigmatique. Au fond, qui ou quoi était Fantômas?

2 Certes il y a des réponses faciles ou évidentes. Fantômas est le Maître de l'Effroi, le Génie du Mal, l'Empereur du Crime, la création de Pierre Souvestre et Marcel Allain qui a fait peur à toute une génération, passant à travers Paris, la France et le monde entier en trente-deux romans au moment du crépuscule de la Belle Époque, de 1911 à 1913. Mais il est aussi évident que Fantômas n'était pas un phénomène sui generis. Il avait des ancêtres, historiques ou fictifs, aussi fascinants que terrifiants et populaires : on peut mentionner les légendes monstrueuses de Robert le Diable et de Gilles de Rais au Moyen Âge, les bandits Cartouche et Mandrin sous l'Ancien Régime, les affaires effroyables de Fualdès et de Lacenaire au début du $19^{\mathrm{e}}$ siècle, des héros criminels du roman-feuilleton du Second Empire, comme Rocambole et les Habits Noirs, l'anarchiste Ravachol et la bande à Bonnot à l'époque des attentats de la fin du $19^{\mathrm{e}}$ et du début du $20^{\mathrm{e}}$ siècle. Même à son époque Fantômas n'était pas le seul héros criminel puissant. Il est l'un des "Terribles" populaires ${ }^{1}$ avec l'Arsène Lupin de Maurice Leblanc, le Chéri-Bibi de Gaston Leroux et le Zigomar de Léon Sazie. Les confrères de Fantômas constituent une 
grande famille épouvantable. Et après? Il y en a d'autres qui sont devenus soit les héritiers, soit les adversaires de Fantômas.

Et il y en a qui l'ont rencontré quelque part, d'une manière ou d'une autre, comme celles et ceux qui assurent la diversité de ce numéro de Belphégor. Mais avant la lecture des articles, il faut dire quelques mots de ceux qui nous ont précédés.

Nous devons mentionner tout d'abord le nom de Francis Lacassin qui a joué un rôle décisif dans l'édition de la série, tout comme il y a consacré plusieurs articles et préfaces et même un dictionnaire des personnages de Fantômas ${ }^{2}$ (n'oublions pas non plus les livres qu'il a écrits sur Louis Feuillade ${ }^{3}$ ). Il faut également penser à Alfu, qui, après avoir publié une première monographie sur cet ensemble romanesque en $1981^{4}$, a également dirigé un numéro de la revue Rocambole pour célébrer le centenaire du Génie du crime ${ }^{5}$.

5 Depuis les années 1960 plusieurs ouvrages ont été consacrés à la série et aux adaptations cinématographiques : « Fantômas ? ... c'est Marcel Allain, le poète masqué, le numéro d'Europe consacré à " Fantômas " ${ }^{7}$, la Nouvelle revue des études fantômassiennes $n^{\circ} 1$ (1993), "Feuillade and the French Serial ${ }^{8}$, "The Lament of Fantômas: The Popular Novel as Modern Mythology ", ou encore Monica dall'Asta et al., Fantômas : la vita plurale di un antieroe (2004).

6 C'est dans cette série d'ouvrages que s'inscrit ce numéro de Belphégor, la revue de l'Association internationale des chercheurs en Littératures Populaires et Cultures Médiatiques (LPCM) : parmi celles et ceux qui ont accepté, cette fois, le défi d'en découdre avec Fantômas, plusieurs sont des membres de cette association. Les auteurs de ce dossier abordent la série dans le contexte de la culture de masse, en mettant l'accent sur l'extrême mobilité du récit, tant au niveau des supports, qu'à celui des cultures : le récit de grande consommation qu'est Fantômas a rapidement produit une nouvelle mythologie.

\section{Le moment Fantômas}

7 1911, l'arrivée de Fantômas sur la scène française (et bientôt internationale) est un moment intense du point de vue de la prolifération des genres et des supports de la littérature. En 1902, les traductions de Conan Doyle paraissent en France ; les fascicules distribués en Europe par Eichler popularisent les aventures de Nick Carter et d'autres. C'est aussi le moment de l'entrée en scène de romanciers comme Gaston Leroux, Gustave Le Rouge ou Maurice Leblanc qui créent, tous, des héros qui restent gravés dans la mémoire collective, soit des enquêteurs comme Rouletabille, soit des méchants comme le docteur Cornélius, soit des enquêteurs-criminels comme Arsène Lupin. L'autonomisation croissante du récit policier va de pair avec le recul du feuilleton et l'importance grandissante des fascicules et des collections populaires (Fayard, Tallandier, Ferenczi). Pour les études paralittéraires, ces nouveaux supports impliquent des changements structurels et thématiques, ce qui exige l'étude minutieuse des paratextes éditoriaux - comme la couverture - à côté de l'étude, plus traditionnelle, des paratextes auctoriaux.

8 Les articles regroupés dans la première partie de ce numéro s'intéressent à ce moment. Charles Grivel n'hésite pas à faire, à la fin de son article-préface, un aveu qui explique son intérêt pour Fantômas. Dans son optique, l'Insaisissable se trouve aux côtés du 
docteur Faustroll : sur la voie de la Pataphysique, Fantômas «n'exprime rien d'autre que le passage à la limite d'un imaginaire qui se joue de sa propre définition.» Fantômas est «illisible ", n'ayant aucune identité, portant masque sous les masques. Il s'agit là d'une idéologie anarchisante, certes, comme on l'a souvent souligné à propos de l'œuvre, mais d'abord pataphysique, parce que le «démenti infligé par le conte au réel [...] engendre moins la crainte que la satisfaction rieuse et le plaisir désentravé ».

L'article de Matthieu Letourneux s'intéresse aux contraintes contextuelles pour démontrer l'originalité des romans de Souvestre et Allain. Selon l'argument de l'auteur, la collection à bon marché de Fayard induit des mutations dans les structures, les imaginaires et les logiques architextuelles des œuvres. Fantômas, directement conçu pour la collection, est marqué à la fois par l'héritage du feuilleton et les contraintes des nouveaux supports. Il en résulte, selon l'auteur, un pacte de lecture sériel fondé sur le rapport des invariants et des variations. La répétition des mêmes structures et la mise en scène des mêmes personnages produisent un sentiment d'irréalité et contraignent les auteurs « à de nouvelles trouvailles, jusqu'à la surenchère ».

L'article d'Isabelle Casta étudie les romans de Fantômas en les situant dans le contexte des « romans de la vitesse ». L'écriture rassemble ainsi, dans la vitesse de sa diégèse, différents états de monde, «tout concourt à faire se téléscoper des habitus réfractaires, dont la rencontre risquée a souvent en effet la teneur surréaliste. » L'auteur - en s'appuyant sur quelques romans de la série - analyse également l'affrontement père/ fille et l'affrontement caïnique. Elle met aussi l'accent sur une hésitation générique propre au roman-feuilleton; c'est ainsi qu'on peut voir, avec cette série, le surgissement d'une sorte de fantasy: flots de sang, cadavres, monologues intérieurs sardoniques, résurrections.

11 Il y a une parenté entre l'article de Charles Grivel et celui de Paul Bleton, puisqu'ils affirment l'un comme l'autre la possibilité d'une lecture autoréférentielle du récit paralittéraire : un Fantômas comme texte illisible, un Zigomar comme cause et comme effet d'impression. Paul Bleton se propose d'étudier ce "compère» de Fantômas, Zigomar, l'homme en cagoule inventé par Léon Sazie, qui est aussi un "compétiteur " qu'il faut dépasser, faire oublier. L'article part de l'étude du contexte littéraire et culturel pour arriver, comme le titre l'indique, aux impressions de Zigomar, et plus particulièrement à celles que la lecture de Zigomar au service de l'Allemagne peut provoquer. Sazie «ne semble avoir qu'assez peu profité de la convergence des fictions populaires imprimées et filmées ", il reste un romancier d'un seul livre. Paul Bleton étudie les diverses publications de Zigomar, les images qui illustrent les éditions, la structure narrative, les personnages et leur fonction pour arriver à cette partie du roman, étape importante dans la formation du roman d'espionnage, qui lie ensemble sérialité et spionspiel.

Annabel Audureau, auteur d'un livre sur Fantômas ${ }^{10}$, poursuit cette fois une recherche entamée dans un des chapitres de son livre: l'étude des couvertures de la série dessinées par Gino Starace. Elle attire l'attention sur l'interaction entre l'illustration et les paratextes, et met en évidence, qu'outre le fait d'assurer une fonction publicitaire, les couvertures de Starace, par leur expressivité, leur dynamisme et leur tension vers un hors-cadre, témoignent d'un véritable souci artistique. Comme l'article le démontre, les 32 couvertures de la série, assurant une lisibilité immédiate, inscrivent Fantômas dans l'imaginaire collectif et contribuent à la construction du mythe. 


\section{Fantômas dans le siècle}

13 Fantômas commence tout de suite à voyager - pour faire référence à l'article de Dominique Kalifa qui se trouve en tête de cette partie. À partir du début du $20^{\mathrm{e}}$ siècle, la culture de masse devient de plus en plus un phénomène mondial et dans ce processus, les nouveaux médias de masse, comme le cinéma ou la radio, jouent un rôle important. Fantômas profite pleinement de cette dynamique. La série est reprise par d'autres supports (cinéma, texte radiophonique, bande-dessinée...) et plusieurs types de relations s'installent entre eux et le texte imprimé de la collection populaire (adaptation, réécriture...). En même temps, on constate également l'intensité de la réception critique.

Les conditions de la circulation transnationale et transmédiatique sont prêtes: Fantômas ne cesse de revenir, il est repris ou commenté de plusieurs façons. C'est un véritable récit de grande consommation proposant toute une nouvelle mythologie.

L'article de Dominique Kalifa, dont la première version a été présentée dans une réunion du Collège de Pataphysique, reprend, à sa manière, les articles de Jean-Claude Vareille ${ }^{11}$ et Maurice Dubourg ${ }^{12}$ consacrés aux voyages de Fantômas. L'auteur précise que - même si Fantômas se rend au Mexique - il y a un lieu, sur le continent américain, les États-Unis, dont l'Insaisissable n'a pas entrepris la conquête. Ce sont donc les héritiers américains qui ont relevé le défi : l'article de Dominique Kalifa donne un bref aperçu de cette conquête.

16 L'article de Nadja Cohen étudie la réception de Fantômas chez les poètes des années 1910 et 1920. Le Génie du Crime apparaît cette fois comme un dandy de type nouveau, énergique, immoraliste par rapport au dandy baudelairien. Fantômas incarne "l'homme moderne", devenant ainsi une figure-clé de l'imaginaire collectif. La célébration de ce personnage subversif est liée à la très forte crise idéologique autour de la Première Guerre.

Bérengère Vachonfrance-Levet, à son tour, propose une analyse détaillée de l'adaptation de Louis Feuillade tirée du premier roman de la série. Fantômas - À l'ombre de la guillotine doit être considéré comme un moment important de l'émancipation du cinéma. À travers l'analyse de l'affiche, de l'encart publicitaire - qui, déjà, indiquent la posture du réalisateur -, de la linéarisation de la narration et de la création de l'univers fantômassien (y compris le problème de la représentation du personnage), cet article précise le rôle du film dans l'évolution du cinéma. À part l'étude du rapport entre littérature, théâtre et cinéma, Bérengère Vachonfrance-Levet met également en avant l'importance de la censure dans la démarche créatrice du réalisateur.

Federico Pagello s'intéresse aux adaptations transnationales et transmédiatiques de Fantômas au début du $20^{\mathrm{e}}$ siècle en les comparant à celles de ses confrères (Raffles d'Ernest W. Hornung, Arsène Lupin de Maurice Leblanc et Zigomar de Léon Sazie). Si on considère les traductions, les adaptations théâtrales ou cinématographiques, seul Arsène Lupin a eu une diffusion internationale plus large que Fantômas. En revanche, à l'échelle mondiale, il semble que les héritiers de Fantômas sont plus nombreux que ses rivaux.

Dans le sillage de ces articles qui s'intéressent aux adaptations cinématographiques, celui de Loïc Artiaga, qui s'appuie sur le dépouillement d'archives inédites, met en avant, lui aussi, l'exigence de l'étude de la plasticité de ces adaptations : c'est ce qu'on a 
oublié jusqu'ici quand on a abordé les trois films réalisés par André Hunnebelle. Loïc Artiaga s'attache ainsi à éclairer la genèse de cette renaissance, la manière dont les années 1960 s'infiltrent dans la trilogie (par exemple à travers la figure de Juve incarné par Louis de Funès) pour arriver, à la fin de son étude, au procès qui oppose l'auteur, Marcel Allain, à la société de productions cinématographiques.

Marie-Alexandra Barataud s'attache à analyser dans son article un curieux «livreobjet » : Fantomas contra los vampiros multinacionales de Julio Cortazár, paru en 1975, à la fois réécriture et adaptation d'une bande dessinée mexicaine, qui avait elle-même adapté l'œuvre française. Après la reconstruction de la mobilité géographique du récit, l'auteur de l'article questionne avant tout le statut générique de cette œuvre exceptionnelle : la rencontre entre plusieurs supports, écritures et personnages permet et nourrit l'expérimentation littéraire.

\section{Variations fantômassiennes}

21 Dans la dernière partie de ce dossier consacré à Fantômas, on trouve deux articles. L'écrivain David White partage avec les lecteurs de précieuses informations sur le sérial américain perdu, réalisé au début des années 20 , lequel offre une adaptation, comme on verra, assez libre de l'œuvre de Souvestre et Allain, écrite et réalisée par Edward Sedgwick. Ce sérial, contentant 20 épisodes, a dû être retitré lors de sa projection en France, suite aux protestations de Marcel Allain. Le public n'a pu voir qu'une version écourtée (12 chapitres) des Exploits de Diabolos. Les sources découvertes par David White, auteur d'une novélisation de cette sérial intitulée Fantômas in America (2007, Black Coat Press), lui ont permis de reconstituer l'intrigue des cinq premiers épisodes.

Les rédacteurs de ce dossier ont eu l'intention d'explorer l'univers de Fantômas dans toutes ses formes, à la recherche de ses héritiers. Une certaine Fantômette peut en être une, quoique un peu lointaine. Dans son article, Christine Leroy offre ainsi une intéressante comparaison, entre Fantômas et ce personnage de la Bibliothèque Rose, né en 1961, Fantômette. L'article, qui passe en revue les traits de caractère majeurs de l'héroïne, met également en évidence qu'il y a peut-être plus de ressemblances entre les deux personnages masqués qu'on ne le pense. Dans les deux cas on voit fonctionner une stratégie d'émancipation: le cas de Fantômette présente, derrière une apparente conformité aux lois, un modèle d'émancipation féminine.

Les rédacteurs de ce dossier vous souhaitent bonne lecture et déclineront toute responsabilité si Fantômas disparaît avec les articles...

\section{NOTES}

1. Antoinette Peské et ïerre Marty, Les Terribles, Paris, Frédéric Chambriand, « Visages », 1951.

2. In Fantômas , volume III, Paris, Robert Laffont, « Bouquins », 1988.

3. F. Lacassin, Maître des lions et des Vampires - Louis Feuillade, Bordas et fils, 1995. 
4. Alfu, L’Encyclopédie de Fantômas, Amiens, autoédition, 1981.

5. « Fantômas centenaire ", Le Rocambole, no. 54, printemps 2011.

6. La Tour de feu, $\mathrm{n}^{\circ} 88,(1965)$.

7. Europe, revue littéraire nos. 590-591 (1978).

8. The Velvet Light Trap: A Critical Journal of Film and Television no. 37 (1996).

9. In Robin Walz, Pulp Surrealism: Insolent Popular Culture in Early Twentieth-Century Paris (2000),

10. Fantômas, Un mythe moderne au croisement des arts, Presses Universitaires de Rennes, 2010.

11. Jean-Claude Vareille, «Le Paris de Fantomas (du pittoresque a l'inquiétant)», Nouvelle revuedes études fantomassiennes, $n^{\circ}$ 1, Paris, Joelle Losfeld, 1993, 69-94.

12. Maurice Dubourg, « Fantômas voyage », Fantômas, Europe, juin-juillet 1978, 590-591.

\section{INDEX}

Mots-clés : Fantômas, littérature populaire, culture médiatique, Souvestre Pierre, Allain Marcel

\section{AUTEURS}

\section{ROBIN WALZ}

Robin Walz est professeur d'histoire à l'Université de l'Alaska (Juneau). Il est l'auteur de Pulp Surrealism : Insolent Popular Culture in Early Twentieth-Century Paris (University of California Press, 2000) et de Modernism, 2ème édition (Pearson, 2013). Spécialiste de l'histoire intellectuelle et de la culture populaire en France, il a écrit des essais sur Fantômas de Pierre Souvestre et Marcel Allain, les Mémoires de Vidocq, Rocambole de Ponson du Terrail, Les Habits noirs de Paul Féval, le détective privé Eugène Villiod, et il a fait traduire en anglais la « Mort du Nick Carter » de Philippe Soupault dans McSweeney's Quarterly 24 (2007). Actuellement il est en train d'écrire un manuscrit sur les « Détectives intrépides, criminels élégants, et justiciers noirs : l'histoire du crime en France, 1815-1950 »

\section{SÁNDOR KALAI}

Sándor Kálai enseigne à l'Université de Debrecen (Hongrie), au Département des Sciences de la Communication et des Médias et au Département des Études Françaises. Il a consacré une thèse, publiée en 2009 et inédite en français, à l'analyse des discours religieux et scientifique dans l'œuvre d'Émile Zola à travers les personnages de prêtre et de médecin qui y évoluent. Outre ses recherches portant sur les œuvres respectives de Zola et d'Octave Mirbeau, au cours de ces dernières années il s'est intéressé à la question du développement de la culture médiatique en Europe. Au sein de ce vaste domaine, ses intérêts se portent tout particulièrement vers le roman policier, de Fortuné du Boisgobey à Thierry Jonquet en passant par Georges Simenon ou JeanPatrick Manchette, au sujet desquels il a consacré différents articles. En 2012 il a publié un livre, en hongrois, sur l'histoire du roman policier français (Fejezetek a francia bünügyi irodalom történetéból, Debrecen, Debrecen University Press) 